\title{
The emergence of computational legal studies: an introduction
}

\section{Ryan Whalen}

This volume arrives at an important inflection point in the relationship between law and computation. Technological, scientific, and methodological developments are increasingly allowing computation to provide not just efficiencies in the traditional ways we practice or study the law, but new perspectives on the law and potential paradigmatic shifts in how we think about and understand it. These developments have already been major factors in the recent evolution of many other academic fields, as evidenced for example by the rise of computational social science, computational biology, the digital humanities, and many more emerged and still-emerging subdisciplines. Although law has perhaps lagged somewhat behind its peer disciplines in adopting and adapting computational research methods, that has begun to change in recent years as more and more legal scholars have begun applying computational methods in the course of their research. This volume explores this emergence of computational legal studies by presenting a variety of research that is either representative of, or in conversation with, the field.

Before setting out to explore the state of computational legal studies, it is important first to provide at least a general delineation of what it might include and exclude. Computers and the law intersect in a variety of ways, none of which is entirely independent of one another, but all of which benefit from being independently identified in a discussion about computational legal studies. Perhaps the most common association between computers and the law is the substantive law that is increasingly faced with questions that arise as society becomes more and more digitally mediated. This area of study and practice is sometimes referred to as cyberlaw, and extends to a diverse set of legal areas including free expression, cybersecurity, privacy, and more. Although cyberlaw is broad in the legal areas it extends to, its scholarship is united by the central relevance of technology and technologically mediated social behavior to the questions it explores.

The application of technology to the practice of law is a second intersection between computation and law. This area, frequently referred to 
as legal tech, encompasses a variety of increasingly diverse and capable technologies that make the work of those engaging with the legal systemincluding, but not limited to lawyers - more efficient. In recent years, there has been substantial growth in the world of legal tech both in the market as developers and firms develop and deploy new legal technologies, and in the academy as both applied and theoretical research and training in legal tech have become more common in law schools. These developments are important not only because they promise to help train more technologically capable practitioners, but also because they increase the exposure of future researchers to methods and technologies that they might not otherwise have been exposed to.

The third intersection between computation and the law occurs when computational research methods are applied to the law as a subject of study. This area-sometimes referred to as computational legal studies - is related both to the long tradition of empirical legal studies and the burgeoning field of computational social science. Computational legal studies includes a wide variety of research and can focus on any substantive area of law. Some projects may use computational techniques to gather data-for instance, by writing a custom web scraper or by downloading and parsing data from open data sources - others might use machine learning or natural language processing techniques to analyze legal data. What unites computational legal research is the fact that it would not be possible without the power of computation-that is, the power to make easy otherwise difficult calculations, or to make trivial otherwise burdensome tasks.

These three intersections are not distinct, and indeed at times intersect with one another. For instance, one might imagine a piece of empirical cyberlaw scholarship that relies on computational techniques for data gathering and analysis (intersecting cyber law with computational legal studies). Similarly, one might study the substantive legal implications of an emerging legal technology such as smart contracts (intersecting cyberlaw with legal tech). Or alternately a computational legal studies approach to understanding a legal technology (intersecting each of cyberlaw, legal tech, and computational legal studies). Although this volume focuses largely on the third of these intersections - the use of computational techniques to study the law-it also touches on issues related to both legal technology, and substantive questions of how the law affects, and is affected by, technology. The complex ways these various intersections between law and technology are intertwined lead any volume such as this to at times touch on each.

The contributions to this volume are diverse both in substantive content, and in methodological focus. They draw on distinct bodies of literature, 
some growing out of the relatively long tradition of Artificial Intelligence (AI) and Law scholarship, others drawing more inspiration from the burgeoning field of computational social science, while still others evolve out of the legal academy's own response to the demand for innovation in law schools. Despite their diversity, these varied contributions can be roughly grouped into four topical categories: text, data, artificial intelligence, and methods and training.

Text has long been central to the law. Indeed, many of humanity's oldest and most famous texts are legal documents, describing contractual relations or legal codes. Because of the central importance of text to the law, the capacity for computational techniques to provide new perspectives and insight into textual data is of particular interest to computational legal scholars. In his chapter on automating legal text comparisons, Alschner details how technological developments have enabled new perspectives on legal documents. Lawyers, policymakers, and law scholars have long been trained to read documents closely and largely in isolation from other related documents. Alschner shows that "distant reading" can also provide useful insight, and goes on to demonstrate methods for doing so by analyzing many subsequent versions of a large body of regulations. In doing so, the chapter helps demonstrate the utility of automated text comparison techniques to those interested in the law and particularly legal change, while also discussing how those applying these techniques must also be cognizant of their particular strengths and weaknesses.

The law is not the only discipline with a special relationship with text. The humanities, with its own special relationship to text, has responded to the computational turn by developing a robust and active subdiscipline often referred to as the digital humanities. In her chapter on textual analyses Varsava provides an overview of textual analytic techniques that have evolved in the digital humanities, and provides insight into the utility these approaches can bring for legal scholars. Digital humanists have developed a robust toolkit, informed by the questions of interest within the humanities and the sources - often texts - that serve as research subjects. As Varsava points out, much of the computationally enabled research done by computational legal scholars can be thought of as an extension of computational social science. Law, however, shares many aspects in common with the humanities, and thus looking to the digital humanities for inspiration and guidance will be fruitful for many computational legal scholars. Varsava leverages these approaches to demonstrate how using computational techniques to measure and compare writing styles can provide insight into judges and the way they write, reason, and work.

Langford, Behn, and Lie also focus on legal texts in their chapter applying stylometric analyses to the text of international investment 
arbitration decisions. The majority of the work on legal writing styles has focused on the work product of judges, and to a lesser extent lawyers. By demonstrating how stylometric techniques show promise in identifying authorship of arbitral award decisions, Langford et al. provide an important methodological contribution that offers promising perspective into an area of law that has long been notoriously opaque. This chapter also offers an example of how legal text extends well beyond judicial opinions, which have tended to be the dominant subject of study especially for those in common law jurisdictions. Legal texts include not just opinions drafted by judges and their clerks, but also arbitral agreements, patents, treaties, statutes, and many other types of documents that are themselves amenable to computational text analyses.

Mainali, Meier, Ash, and Chen also take a language-centric approach to the study of law. However, their approach focuses not on the style of judicial writing, but rather on the degree to which the author's style of reasoning can be detected via machine learning. This approach highlights the potential for semi-supervised classification techniques to aid in the sorts of legal research that requires analyzing many hundreds, thousands, or even millions of documents. These techniques enable research at a scale not feasible for researchers unassisted by automated classification methods. The emergence and popularization of these methods-along with the rapid growth in the availability of machine-readable judicial opinions - is in the midst of generating a new style of empirical judicial behavior research.

The importance of textual data to computational legal studies has turned many scholars' attention to questions about access to legal texts and the quality and coverage of existing databases. In their chapter demonstrating the data-collection efforts underpinning a research project examining labor law decisions, Alexander and Feizollahi explore these questions about data access and coverage and the implications for computational legal scholars. Data-driven research quite naturally requires data, and if it is to make statistical extrapolations it requires not only access to data, but an understanding of potential sampling biases. Alexander and Feizollahi show that the traditional sources of American judicial opinion data can have dramatically different levels of coverage. As a partial solution, they advocate for courts to produce easily accessible and machine-readable data. Doing so would improve scholars' ability to engage in large-scale data-driven research and help ensure that American courts do not fall too far behind their international peers in this regard.

Much of the existing legal academic research that uses computational research methods is either performed in Western jurisdictions or focuses on Western legal systems. However, the evolution of the Chinese legal system, and especially its adoption of data-driven decisionmaking, offers 
rich ground for computationally enabled empirical legal studies. In their chapter on the role of computational legal studies within China, Tang and Liu chart the rise of Chinese empirical legal studies, and discuss both the potential for computational approaches to contribute to the development of the Chinese legal academy as well as some of the challenges they foresee along the way. Many of these challenges mirror those facing the emergence of computational legal studies globally, but some are challenges with Chinese characteristics. Regardless of precisely how computationally enabled legal scholarship evolves in China, it is almost certain to become a more important element of that country's academy, and Tang and Liu's contribution offers a valuable overview and discussion of the field for those who would like to better appreciate its current state and future prospects.

The data access revolution is not limited to the increasing availability of legal documents such as judicial opinions. Indeed, as more and more social behavior becomes digitally mediated, the digital traces of that behavior leave more and more data of interest to legal scholars. In his chapter on measuring regulatory impact at scale, Penney demonstrates the implications that the increase in computer mediated social interaction has for empirical legal scholars. As regulation increasingly affects digitally mediated activity, legal scholars are left with an obligation to better understand how these regulations may alter behavior, and are also left with a rich body of data with which to do so. Penney capitalizes on a natural experiment arising from policy disclosure and uses the digital trace data left by Wikipedia editors to convincingly demonstrate the power of his approach in furthering our empirical understanding of how regulation affects behavior. In doing so, he shows how the legal issues, such as the disclosure of US Government surveillance activities, can manifest in changed online activity that can be detected and measured with the use of computational techniques.

Suzor's chapter on understanding content moderation systems likewise engages with the shift towards increasing digitally mediated social behavior. As this has occurred, individuals have not only become more likely to have their online behavior affected by official regulations and policies - as evidenced in Penney's contribution - but also to be subject to regulation by online platforms themselves. The regulation by platform is potentially just as important as state-sponsored laws and regulations in affecting social behavior. As such, understanding how it manifests is a crucially important task for contemporary legal scholars. However, as private enterprises, online platforms are not bound by the same expectations of transparency as public actors are. Suzor's contribution is to detail a sophisticated data gathering project that allows researchers to track and 
better understand content moderation at scale. This is an example of what one might call a "data infrastructure" project that facilitates future work and improves the capacity of computational legal studies to contribute to emerging policy debates.

Following the contributions focused on text analysis and data, the volume then turns to one of the highest visibility areas of contemporary computational legal studies - artificial intelligence and machine learning. As access to data has increased, and computational methods have become more sophisticated and capable, the capacity for machine-learning models to mimic human intelligence has improved. This has raised the profile of legal artificial intelligence, with increased focus both on practical aspects of how to develop and deploy these technologies as well as more theoretical questions about what AI may mean for the practice of law and whether indeed there are some elements of legal systems that are so human-centric that they are not amenable to automated decisionmaking.

In a chapter discussing whether artificial intelligence can be designed to account for complexity, Ashley demonstrates how AI models can be specified to consider not just questions of law and fact, but to also account for social values. Doing so provides an empirical perspective on values-based reasoning that will be of interest not only to designers and consumers of legal AI models, but also to those interested in the role that values play in legal reasoning. In doing so, Ashley's contribution demonstrates the capacity for computational legal studies to engage simultaneously with practical questions facing the development of legal AI and more fundamental questions of jurisprudence.

These fundamental questions include those that computation and computational research methods raise about how technological development might affect legal decisionmaking and ultimately the law itself. Gowder takes up this line of questioning at the intersection between computation and law by asking whether legal cognition is computational. Using a classic legal classification problem to demonstrate, Gowder shows how modern machine-learning methods could be used as decisionmaking aides to help answer a subset of legal questions. By demonstrating the potential for computation as a judicial aide, Gowder's contribution shows how computational approaches could conceivably influence the future development of how courts function, while also reminding us of their limitations.

Livermore also takes up questions at the very heart of law and computation by exploring the implications of a legal system adopting machinelearning decisionmaking tools. In doing so, Livermore highlights both the potential strengths and weaknesses of computational decisionmaking technologies, while also raising important questions about the degree 
to which getting the right answer is as important as legal process, and whether or not machines can be meaningful participants in legitimate legal systems. These questions that Livermore tackles are of fundamental importance to those who foresee or advocate for an increasing reliance on technology in legal systems. As those technologies become more capable, and more widely adopted, better understanding the degree to which lawas-computation risks impinging upon law's essential humanity and its ability to evolve in step with the societies it rules is imperative.

Following its exploration of the intersection of computational law and jurisprudence, the volume's final section turns to specific computational legal studies methods, applications, and education. Among the varied methods at the disposal of computational legal scholars, network analysis has shown promise in providing a structural and system-level perspective that is quite distinct from traditional legal scholarship methods. In their chapter on citation network analyses of case law, van Kuppevelt, van Dijck, and Schaper provide an overview of this increasingly popular area of research. In doing so, they detail methodological developments required to ensure that legal citation network analyses are sufficiently rigorous, reproducible, and interpretable. As a relatively new area of inquiry, this detailed overview and comparison of various network analysis methods and sampling strategies provides an important cautionary tale to researchers, demonstrating the importance of the research design decisions they make.

Following van Kuppevelt et al.'s overview of legal network analyses, Olsen and Esmark provide an applied demonstration of the utility that a network perspective can provide. After mapping citations to case law from the European Court of Human Rights, they use centrality measures to identify those cases containing general principles of law, as opposed to those related to more narrow or specific legal questions. Doing so demonstrates the potential of a network-driven approach to make sense of case law in ways that traditional legal scholarship would not, and thereby exemplifies the way a computational perspective can provide a unique quality of insight into the law.

Network analysis is just one of a wide variety of techniques at the disposal of computational legal scholars. Schwartz introduces another in his chapter where he provides an overview of agent-based simulations, and subsequently a demonstration of how they can be applied to questions of interest to legal scholars. Schwartz shows how agent-based models can be used to provide insight into aspects of legal systems that are otherwise difficult to study. By simulating the behavior of legal actors - in this chapter's case, the actions of courts and government agents-agent-based models are able to play out various scenarios and demonstrate how system-level 
effects like the emergence of a stable high court, or the failure of the rule of law, can be affected by agent-level behavior. Social simulations have proven very insightful throughout the social sciences, and Schwartz shows in his research that this utility also extends to the study of the law.

In the volume's final chapter Beckedorf, Hartung, and Sittig discuss an area of central importance to the emergence of computational legal studies - that is, integrating computational training into the law school curriculum. Their chapter demonstrates the potential of hands-on interdisciplinary training to empower teams of law and computer science students to engage in meaningful computationally enabled legal research. In doing so, they highlight how their educational program, and others like it, are facilitating the emergence of computational legal studies. Training programs are central to the development of academic subfields and methodological camps. Traditional legal training models have perhaps lagged behind others in providing avenues through which their graduates can become proficient in empirical and computational research techniques. Programs such as that highlighted by Beckedorf et al. will not only help train computationally literate practitioners but also researchers and academics.

Taken together the contributions in this volume sketch out many of the promises of computational legal studies in furthering our understanding of the law and sociolegal systems. Meanwhile, this collection also helps identify the challenges facing computational legal scholars and students as they seek to further establish the field, its methods, and how it engages with a wider community of scholars, policymakers, and practitioners. Although these challenges are substantial, the field's promise - coupled with the rapid growth in computing power, data access, research methods, and trained researchers-suggests that computational legal studies will thrive as it continues to provide its unique perspective on the law. 